\title{
Improving patient safety after rigid bronchoscopy in adults: laryngeal mask airway versus face mask - a pilot study
}

This article was published in the following Dove Press journal:

Medical Devices: Evidence and Research

30 April 2015

Number of times this article has been viewed

\author{
Fulvio Nisi' \\ Antonio Galzerano' \\ Gaetano Cicchitto ${ }^{2}$ \\ Francesco Puma ${ }^{3}$ \\ Vito Aldo Peduto' \\ 'Department of Anesthesiology, \\ Intensive Care and Pain Therapy \\ Centre, ${ }^{2}$ Department of Pneumology \\ and Respiratory Medicine, \\ ${ }^{3}$ Department of Thoracic Surgery, \\ AO Santa Maria della Misericordia \\ Perugia, Italy
}

Background: There are still no clear guidelines in the literature on per procedural bronchoscopic management for anesthesiologists, and few relevant datasets are available. To obtain rapid recovery from anesthesia, it is often necessary to keep patients in the recovery room for several hours until they become clinically stable. In this study, we tested the hypothesis that the laryngeal mask airway (LMA) enables better respiratory and hemodynamic recovery than the oxygen face mask (FM) in patients undergoing rigid bronchoscopy.

Methods: Twenty-one patients undergoing elective bronchoscopy of the upper airway were randomized to ventilation assistance with FM or LMA after a rigid bronchoscopy procedure under general anesthesia. The primary endpoint was duration of post-surgical recovery and the secondary endpoints were postoperative hemodynamic and respiratory parameters. Assessment of the study endpoints was performed by an intensive care specialist blinded to the method of ventilation used. The statistical analysis was performed using the Fisher's Exact test for nominal data and the Student's $t$-test for continuous data.

Results: There was no statistically significant difference in post-procedural time between the two groups $(P=0.972)$. The recovery parameters were significantly better in the LMA group than in the FM group, with significantly fewer desaturation, hypotensive, and bradycardic events $(P<0.05)$.

Conclusion: We conclude that the LMA may be safer and more comfortable than the FM in patients undergoing rigid bronchoscopy.

Keywords: face mask, laryngeal mask airway, anesthesiology, rigid bronchoscopy, safety

\section{Introduction}

The advent of bronchoscopy has profoundly changed modern respiratory medicine. More than $98 \%$ of bronchoscopies are carried out with a flexible bronchoscope, ${ }^{1}$ and most specialists in bronchoscopy have never had training to improve their technique when performing rigid bronchoscopy. For specialists in anesthesiology, rigid bronchoscopy remains a "ventilation challenge" owing to the intrinsic nature of the surgical procedure, which takes place in the same channel through which the anesthesiologist is trying to ventilate the patient. In some aspects, postoperative assistance could influence patient outcome, but above all it enables more rapid hemodynamic and respiratory recovery by ensuring successful and efficient airway control with adequate oxygenation-ventilation flows. Ideally, this should be achieved with the minimum of patient discomfort.

There are still no clear guidelines in the literature on per procedural bronchoscopic management for anesthesiologists, and few relevant datasets are available.
Department of Anesthesiology, Intensive Care and Pain Therapy Centre, AO Santa Maria della Misericordia, via G Dottori, 06126 Perugia, Italy

Tel +39392598 I22I

Email fulvio.nisi@gmail.com 
Consequently, to allow rapid recovery from anesthesia, it is often necessary to keep patients in the recovery room for several hours until their clinical condition stabilizes.

Ventilating via preoxygenation and apneic ventilation make it possible to maintain spontaneous breathing during a bronchoscopic procedure, and the use of a face mask (FM) guarantees rapid recovery and patient comfort in the postoperative period. However, even if the FM is relatively safe, it requires a period of time to establish effective ventilation, and there is the possibility of temporary desaturations if management of the airways is difficult, ${ }^{2}$ although not all authors agree with this view. ${ }^{3,4}$ On the other hand, the laryngeal mask airway (LMA) enables successful ventilation and can measure tidal volume and gas flow, ${ }^{5-7}$ even in patients who are difficult to ventilate with an $\mathrm{FM}^{8}{ }^{8}$ The main disadvantages of the LMA is the need for deeper analgesia and sedation to allow straightforward insertion of the device with minimal discomfort for the patient. ${ }^{9,10}$ With regard to safety, the factors that most strongly influence choice of device are the duration of postoperative assistance required and complications. Postoperative assistance starts immediately after removal of the bronchoscope from the airways and continues in the recovery room until hemodynamic and respiratory stabilization is complete.

Post-anesthesia care in the recovery room contributes significantly to the reduction in risk of postoperative complications. The purposes of the recovery room include reversal of the pharmacological effects of general anesthesia, stabilization of respiratory and hemodynamic parameters, and intensive intervention in the event of acute complications. The main complications occurring in the recovery room are respiratory (airways obstruction, hypoxemia, hypoventilation, inhalation), cardiovascular (hypotension, hypertension, arrhythmia, myocardial ischemia), postoperative nausea and vomiting, hypothermia and hyperthermia, delayed reawakening, disorientation and hyperexcitability, and postoperative shivering. Before the patient can be discharged from the recovery room, the following requisites must be satisfied: return of a state of consciousness, stable cardiocirculatory parameters, absence of respiratory depression, absence of bleeding, absence of nausea and vomiting, good analgesia, and recovery of movement in the event of locoregional anesthesia. Thus, accurate monitoring in the recovery room is essential for postoperative safety. ${ }^{11}$

We tested the hypothesis that the LMA is safer and allows better respiratory and hemodynamic recovery than the oxygen FM in patients undergoing rigid bronchoscopy. The primary endpoint was duration of postoperative recovery and the secondary endpoints were the amounts of ventilatory and hemodynamic support required in patients undergoing rigid bronchoscopy, according to whether they received the LMA or the oxygen FM.

\section{Patients and methods}

\section{Patients}

This study involving human subjects was conducted in accordance with tenets of the Declaration of Helsinki (1975) as revised in 2000. After approval of the study design by the institutional ethics committee, informed consent was obtained from each patient before entry to the study.

Patients were recruited according to the following inclusion criteria: elective interventional rigid bronchoscopy; mechanical or functional tracheal obstruction (tracheal stenosis, neoplasm, bronchopleural fistula, foreign body, other type of airway obstruction); duration of procedure $\leq 2$ hours; and American Society of Anesthesiologists (ASA) score I-III. Postoperatively, the patients were randomly assigned to an FM group or to an LMA group.

Exclusion criteria were: age $<18$ years; emergency procedures and/or need for emergency airway management (mini-tracheostomy); endoscopic procedure other than rigid bronchoscopy; a diagnostic procedure performed via rigid bronchoscopy; requirement for endotracheal intubation after rigid bronchoscopy; single lung ventilation, thoracotomy, or lobectomy before/after rigid bronchoscopy; procedure lasting longer than 2 hours; and an ASA score of IV-V. Thus, patients undergoing intensive procedures lasting more than 2 hours were excluded, because of the difficulties involved in assessment of what kind of airway management was performed. The rationale was to standardize the management of anesthesia-related airway complications.

\section{Protocol and monitoring}

We used a 7.5 or $8.5 \mathrm{~mm}$ Storz ventilating rigid bronchoscope with a standardized protocol for drug administration. Atropine $0.5-1 \mathrm{mg}$ was used to reduce secretions in the airways and to suppress parasympathetic reflexes, and midazolam 1-2 mg was used to achieve optimal sedation. All patients were preoxygenated via a Magill circuit with a fraction of inspired oxygen of $100 \%$ for 3-5 minutes before the start of the procedure. Lidocaine $2 \%$ was applied to the oropharynx and larynx as topical anesthesia. Continuous total intravenous anesthesia (propofol $2 \mathrm{mg} / \mathrm{kg} / \mathrm{h}$ and remifentanil $0.05 \mu \mathrm{g} / \mathrm{kg} / \mathrm{min}$ ) was used to maintain anesthesia.

After introduction of the bronchoscope, the side arm of the instrument was linked to a Magill circuit allowing 
both spontaneous and assisted ventilation. Manual assisted ventilation was used in the critical phase of the procedure after induction and before control of the airway with the rigid bronchoscope. We assisted the patient until adequate spontaneous ventilation was recovered. Post-procedural ventilatory assistance was provided by the FM or LMA in the recovery room. Patients were not discharged from the recovery room until achievement of the following goals: return of consciousness, stable cardiocirculatory parameters, absence of respiratory depression, absence of bleeding, absence of nausea and vomiting, good analgesia, and recovery of spontaneous movements.

\section{Methods}

We recorded the duration of the surgical procedure and the duration of postoperative ventilatory assistance (from the end of the surgical procedure until hemodynamic and respiratory stabilization) for each group. We also recorded the total amounts of opioids and hypnotic agents administered.

Per procedural complications of rigid bronchoscopy reported in the literature ${ }^{12,13}$ include those directly related to the surgical technique (mechanical trauma to the teeth, hemorrhage, bronchospasm, bronchial or tracheal perforation) and those related to anesthetic management (hypoxemia, glottic edema, extrasystoles and arrhythmias, barotrauma). We selected arterial hypertension/hypotension, bradycardia, and tachycardia (believed to increase the risk of arrhythmias) as hemodynamic parameters and desaturation as the respiratory parameter, because of their relationship with anesthetic management. Oxygen saturation, systolic blood pressure, and heart rate were recorded for each procedure. With regard to the hemodynamic parameters, we considered the number of patients who experienced at least one episode of systemic arterial hypertension (a $>20 \%$ increase in systolic arterial pressure from baseline), hypotension (a $>20 \%$ decrease in systolic arterial pressure from baseline), tachycardia (heart rate $>100 \mathrm{bpm}$ for more than 3 minutes), or bradycardia (heart rate $<50 \mathrm{bpm}$ for more than 3 minutes). Desaturation was defined as a hypoxemic event where there was a $>5 \%$ decrease in oxygen saturation from the preoperative baseline on pulse oximetry.

\section{Statistical analysis}

The patients were divided into two groups by computerized randomization (Randomization.com). The Student's $t$-test was used to compare the time spent in postoperative recovery and rates of hemodynamic and respiratory complications between the two groups. $P<0.05$ were considered to be statistically significant. The statistical analysis was performed using GraphPad Prism software version 6.02 for Mac (GraphPad Software, La Jolla, CA, USA). The study endpoints was measured by an intensive care specialist blinded to the type of ventilatory assistance received.

\section{Results}

Thirty-two patients undergoing rigid bronchoscopy were recruited for this study between January 2012 and May 2013. Four patients were excluded because of a need for intubation after their procedure, four required prolonged sedation for postoperative complications, and three needed mini-tracheostomy for emergency airway management, giving a final study population of 21 patients.

The mean age of the patients was $64 \pm 4.2$ years, with $71.4 \%$ being women and $28.6 \%$ being men (Table 1 ). The diagnoses on admission were: non-neoplastic tracheal or laryngotracheal stenosis (33.3\%); malignant tracheobronchial stenosis (carcinoma or carcinoid tumor, 38.0\%); benign tracheobronchial tumor (tracheal papillomatosis, 14.3\%); bronchopleural fistula (4.7\%); and foreign body (9.5\%). Three patients (14.3\%) had an ASA score of I, three (14.3\%) had a score of II, and 15 (71.4\%) had a score of III. No patient showed any features predictive of difficult airway management during preanesthesia examination. The most common comorbidities were hypertension (38.1\%), chronic

Table I Study population

\begin{tabular}{|c|c|c|c|c|}
\hline & $n=21$ & $\begin{array}{l}\text { FM } \\
\text { group } \\
(n=10)\end{array}$ & $\begin{array}{l}\text { LMA } \\
\text { group } \\
(n=I I)\end{array}$ & $P$-value \\
\hline Age, years & $64 \pm 4.2$ & $57 \pm 17.86$ & $70 \pm 12.84$ & 0.561 \\
\hline Sex (male/female) & $6 / 15$ & $3 / 7$ & $3 / 8$ & 0.890 \\
\hline BMI & $28 \pm 2$ & $27 \pm 3$ & $29 \pm 2$ & 0.895 \\
\hline ASA score I-III & 3 & $3 / 1 / 6$ & $2 / 2 / 7$ & 0.887 \\
\hline \multicolumn{5}{|l|}{ Admission surgical diagnosis } \\
\hline Non-neoplastic stenosis & 7 & 4 & 3 & \\
\hline Malignant stenosis & 8 & 4 & 4 & \\
\hline Benign tumors & 3 & 2 & I & \\
\hline Bronchopleural fistula & I & 0 & I & \\
\hline Foreign body & 2 & I & I & \\
\hline \multicolumn{5}{|l|}{ Comorbidities } \\
\hline Hypertension & 8 & 5 & 3 & 0.424 \\
\hline COPD & 7 & 3 & 4 & 0.556 \\
\hline Cardiovascular disease & 8 & 5 & 4 & 0.663 \\
\hline Dyslipidemia & 5 & 3 & 2 & 0.531 \\
\hline
\end{tabular}

Notes: Data are expressed as the mean \pm standard deviation or absolute value. A $P$-value $<0.05$ was considered to be statistically significant.

Abbreviations: ASA, American Society of Anesthesiologists; BMI, body mass index; COPD, chronic obstructive pulmonary disease; FM, face mask; LMA, laryngeal mask airway. 
obstructive pulmonary disease $(33.3 \%)$, cardiovascular disease (38.1\%), and dyslipidemia (23.7\%, Table 1).

Ten patients were randomized to FM ventilation and eleven to LMA ventilation. The two groups were similar with regard to age, sex, ASA score, body mass index, and comorbidities (Fisher's Exact test, Table 1). Operative time and postsurgical ventilatory assistance were also similar between the two groups, as were the doses of remifentanil and propofol per treated patient (unpaired $t$-test, Table 2). Duration of postoperative surgical recovery, the primary endpoint of the study, was not significantly different between the groups

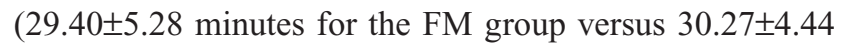
minutes for the LMA group, $P=0.972$, Table 2).

Desaturation was significantly more frequent in the FM group than in the LM group $(P<0.05)$. Similarly, there were significant differences between the groups with regard to number of episodes of arterial hypotension and bradycardia. No differences were noted for other cardiovascular and respiratory parameters (Table 3 ).

\section{Discussion}

To our knowledge, this randomized pilot study is the first investigation of the safety of FM versus LMA for assisted ventilation in postoperative recovery after rigid bronchoscopy. In this context, both FM and LMA required a similar ventilatory assistance time before achievement of hemodynamic and respiratory stability. Therefore, neither FM nor LMA offer an advantage in terms of speed of recovery after rigid bronchoscopy. However, an interesting feature emerged from the safety analysis, ie, hemodynamic and respiratory stability was improved in patients assisted with the LMA. In addition, although not an endpoint of this study, FM failed to provide better comfort than LMA, as suggested by a higher incidence of jaw thrust in patients ventilated by the FM.

The majority of studies relating to rigid bronchoscopy have compared spontaneous breathing with controlled ventilation (including jet ventilation and high frequency ventilation) during a surgical procedure with total intravenous

Table 2 Operative data

\begin{tabular}{llll}
\hline & $\begin{array}{l}\text { FM group } \\
(\mathbf{n}=1 \mathbf{0})\end{array}$ & $\begin{array}{l}\text { LMA group } \\
(\mathbf{n}=1 \mathrm{I})\end{array}$ & $\boldsymbol{P}$-value \\
\hline $\begin{array}{l}\text { Duration of surgery (minutes) } \\
\text { Duration of post-surgical }\end{array}$ & $61.20 \pm 22.45$ & $58.72 \pm 23.73$ & 0.955 \\
support (minutes) & $29.40 \pm 5.28$ & $30.27 \pm 4.44$ & 0.972 \\
$\begin{array}{l}\text { Remifentanil, total dose }(\mu \mathrm{g}) \\
\text { Propofol, total dose }(\mathrm{mg})\end{array}$ & $215 \pm 75$ & $210 \pm 90$ & 0.925 \\
\hline
\end{tabular}

Notes: The data are shown as the mean \pm standard deviation. $P<0.05$ was considered to be statistically significant.

Abbreviations: FM, face mask; LMA, laryngeal mask airway.
Table 3 Hemodynamic and respiratory events during the postoperative period

\begin{tabular}{lllll}
\hline & $\begin{array}{l}\text { FM } \\
\text { group } \\
(\mathbf{n}=\mathbf{1 0})\end{array}$ & $\begin{array}{l}\text { LMA } \\
\text { group } \\
(\mathbf{n}=\mathbf{I I})\end{array}$ & $\mathbf{P}<\mathbf{0 . 0 5}$ & $\mathbf{P}$-value \\
\hline Desaturation & 2 & - & $P<0.05$ & 0.019 \\
Arterial hypertension & $\mathrm{I}$ & $\mathrm{I}$ & $\mathrm{NS}$ & 0.384 \\
Arterial hypotension & 2 & - & $\mathbf{P}<0.05$ & 0.046 \\
Tachycardia & 2 & $\mathrm{I}$ & $\mathrm{NS}$ & 0.175 \\
Bradycardia & 2 & - & $P<0.05$ & 0.038 \\
\hline
\end{tabular}

Notes: Data are expressed as absolute values. See text for definitions of events. Abbreviations: FM, face mask; LMA, laryngeal mask airway; NS, not significant.

anesthesia provided by propofol. Some of these studies suggest that spontaneous ventilation is preferable to controlled ventilation because it provides good analgesia and sedation without respiratory depression. ${ }^{14-16}$ Advantages of spontaneous breathing when compared with jet ventilation also include continuous monitoring of compliance, the ability to estimate tidal volume, achievement of good $\mathrm{PaO}_{2}$ levels in the airways (better than Sander jet ventilation ${ }^{17}$ ), and a minor risk of barotrauma in patients with critical airway stenosis. ${ }^{18,19}$ Nevertheless, spontaneous ventilation could lead to unfavorable blood-gas values during prolonged bronchoscopic procedures; hypercapnia, in particular, with its attendant risk of cardiac arrhythmia.

Despite the wide range of studies concerning anesthetic management during rigid bronchoscopy, procedural safety has always been analyzed perioperatively and has never focused on postoperative management or complications, or how to prevent them. In fact, airway procedures increase the risk of per procedural complications, including hypoxia and arrhythmia, which could be made worse by inappropriate ventilatory support. Choosing the best device is the first step. Interestingly, in the present study, the incidence of postoperative complications was higher in the FM group. Moreover, FM-assisted patients not only had more postoperative desaturation episodes but also had significantly lower percent oxygen saturation. Similarly, the FM group had more hypertensive peaks and bradycardia before recovery of hemodynamic stability.

The duration of postoperative assistance required in the recovery room was not significantly different between the LMA and FM groups, thus pointing out the experience of the thoracic anesthetist using the device. The majority of the recent studies concerning LMA and FM highlight the ability to ventilate the airway more successfully and rapidly using the LMA, providing the anesthetist is skilled in use of the LMA technique, although rapid airway 
control can still be established with FM if the operator is less experienced with use of the LMA. ${ }^{3}$ Therefore, having an experienced anesthetist minimizes any differences between the devices with regard to establishing airways control.

Some limitations to this study should be taken into account. First, postoperative electrocardiography monitoring was not included in the study design, and could have added further information on cardiac adverse events (such as ST elevations) related to the procedure. Although it is still untimely to be considered indicative of cardiac ischemia, ${ }^{20}$ it could be interesting to analyze as parameter of hemodynamics. Unfortunately, the study setting did not allow this kind examination to be extended to all patients during recovery room assistance time.

Second, it should be noted that our choice of opioid (remifentanil) could have possibly made the arise of perioperative depression of respiratory drive or influenced the postoperative recovery. Nevertheless, it could be argued that a short-acting opioid like remifentanil would enable more rapid recovery when compared with the other opioids available, ${ }^{21,22}$ although it should be noted that the risk of opioid-induced respiratory depression is low in these cases.

Third, our study may have lacked the statistical power to draw definite conclusions regarding our secondary endpoint, ie, the fact that more hemodynamic complications occurred in the FM group than in the LMA group. The surgical procedure included in our study is limited to a highly selected group of patients, and even if the study was carried out in a regional multidisciplinary center with a medium-term to long-term observation period, the data collected still relate to a small sample. For this reason, we have presented our work as a pilot study, pending further considerations about it. Finally, we did not include patients undergoing emergency bronchoscopy or critically ill patients with ASA scores of IV-V, so caution should be exercised when extrapolating our study results to these surgical populations.

\section{Conclusion}

Choice of the most appropriate ventilating device continues to be based on the clinical experience of anesthetists specialized in thoracic surgery. This study shows that the LMA prevents desaturation and perioperative hypotension more effectively than the FM. Although probably not having any survival advantage, we believe that the LMA could be considered preferable to the FM for ventilation because of its lower complication rate.

\section{Disclosure}

The authors report no conflicts of interest in this work. This research was presented as a poster communication at the 67th National Congress of the Italian Society of Anaesthesiology and Intensive Care Medicine, October 16-19, 2013, Rome, Italy.

\section{References}

1. Tobin MJ, D'Alonzo GE. Bronchoscopy in intensive care. Appl Cardiopulm Pathophysiol. 1991;3:319-325.

2. Brimacombe J. The advantages of the LMA over the tracheal tube or facemask: a meta-analysis. Can J Anaesth. 1995;42:1017-1023.

3. Blevin AE, McDouall SF, Rechner JA. A comparison of the laryngeal mask airway with the face mask and oropharyngeal airway for manual ventilation by first responders in children. Anaesthesia. 2009;64: 1312-1316.

4. Rechner JA, Loach VJ, Ali MT. A comparison of the laryngeal mask airway with facemask and oropharyngeal airway for manual ventilation by critical care nurses in children. Anesthesia. 2007; 62:790-795.

5. Russo SG, Bollinger M, Strack M. Transfer of airway skills from manikin training to patient: success of ventilation with facemask or LMA-Supreme (TM) by medical students. Anaesthesia. 2013;68: $1124-1131$.

6. Cameron AE, Sievert J, Asbury AJ. Gas leakage and the laryngeal mask airway. A comparison with the tracheal tube and facemask during spontaneous ventilation using a circle breathing system. Anesthesia. 1996;51:1117-1119.

7. Ho-Tai LM, Devitt JH, Noel AG, O'Donnell MP. Gas leak and gastric insufflation during controlled ventilation: face mask versus laryngeal mask airway. Can J Anaesth. 1998;45:206-211.

8. Abdi W, Dhonneur G, Amathieu R. LMA supreme versus facemask ventilation performed by novicies: a comparative study in morbidity obese patients showing difficult ventilation predictors. Obes Surg. 2009;19:1624-1630.

9. Montazari K, Naghibi Kh, Hashemi SJ. Comparison of hemodynamic changes after insertion of laryngeal mask airway, facemask and endotracheal intubation. Acta Med Iran. 2004;42:437-440.

10. Brimacombe J, Holyoake L, Keller C. Pharyngolaryngeal, neck, and jaw discomfort after anesthesia with facemask and laryngeal mask airway at high and low cuff volumes in males and females. Anesthesiology. 2000;93:26-31.

11. Leykin Y, Costa N, Gullo A. Recovery room. Organization and clinical aspects. Minerva Anestesiol. 2001;67:539-554.

12. Miller JI Jr. Rigid bronchoscopy. Chest Surg Clin N Am. 1996;6: 161-167.

13. Wain JC. Rigid bronchoscopy: the value of a venerable procedure. Chest Surg Clin NAm. 2001;11:691-699.

14. Gilles P, Henri G, Colt MD, et al. Safety of interventional rigid bronchoscopy using intravenous anesthesia and spontaneous assisted ventilation A prospective study. Chest. 1992;102:1526-1530.

15. Farrell PT. Rigid bronchoscopy for foreign body removal: anaesthesia and ventilation. Paediatr Anaesth. 2004;14:84-89.

16. Dumon JF, Shapshay S, Borcereau J, et al. Principles for safety in application of neodymium-YAG laser in bronchology. Chest. 1984;86: $163-168$.

17. Giesecke AH Jr, Gerbershagen HU, Dortman C. Comparison of the ventilationg and injection bronchoscopes. Anesthesiology. 1993;38: 298-303.

18. Dutau H, Vandermoortele T, Breen DP. Rigid bronchoscopy. Clin Chest Med. 2013;34:427-435.

19. Jaquette Y, Monnier P, Van Melle G, et al. Complications of different ventilation strategies in endoscopic laryngeal surgery. A 10-year review. Anesthesiology. 2006;104:52-59. 
20. Hill AJ, Feneck RO, Underwood SM, et al. The hemodynamic effects of bronchoscopy. Comparison of propofol and thipentone with and without alfentanil pretreatment. Anesthesia. 1991;46:266-270.

21. Ozkose Z, Yalcin Cok O, Tuncer B. Comparison of hemodynamics, recovery profile, and early postoperative pain control and costs of remifentanil versus alfentanil-based total intravenous anesthesia (TIVA). J Clin Anesth. 2002;14:161-168.
22. Natalini G, Frassini P, Seramondi V, et al. Remifentanil vs fentanyl during interventional rigid bronchoscopy under general anesthesia and spontaneous assisted ventilation. Eur J Anesthesiol. 1999;16: 605-609.

\section{Publish your work in this journal}

Medical Devices: Evidence and Research is an international, peerreviewed, open access journal that focuses on the evidence, technology, research, and expert opinion supporting the use and application of medical devices in the diagnosis, treatment and management of clinical conditions and physiological processes. The identification of novel devices and optimal use of existing devices which will lead to improved clinical outcomes and more effective patient management and safety is a key feature. The manuscript management system is completely online and includes a quick and fair peer-review system. Visit http://www. dovepress.com/testimonials.php to read real quotes from authors.

Submit your manuscript here: http://www.dovepress.com/medical-devices-evidence-and-research-journal 